\title{
Nanofluidic Fluorescence Microscopy (NFM) for real-time monitoring of protein binding kinetics and affinity studies
}

Pattamon Teerapanich ${ }^{\mathrm{a}, \mathrm{b}}$, Martine Pugnière ${ }^{\mathrm{c}, \mathrm{d}}$, Corinne Henriquet ${ }^{\mathrm{c}, \mathrm{d}}$, Yii-Lih Lin ${ }^{\mathrm{e}}$, Chia-Fu Chou ${ }^{\mathrm{e}}$, and Thierry Leïchléa,b,*

aLAAS-CNRS, 7 avenue du Colonel Roche, F-31077 Toulouse, France

bUniversité de Toulouse, F-31077 Toulouse, France

'IRCM, Institut de Recherche en Cancérologie de Montpellier; INSERM, U1194

dUniversité Montpellier; ICM Institut Régional du Cancer, Montpellier, F-34090 France

eInstitute of Physics, Academia Sinica, Taipei 11529, Taiwan

*Corresponding author

Email address: tleichle@laas.fr (T. Leïchlé)

\begin{abstract}
Kinetic monitoring of protein interactions offers insights to their corresponding functions in cellular processes. Surface plasmon resonance (SPR) is the current standard tool used for label-free kinetic assays; however, costly and sophisticated setups are required, decreasing its accessibility to research laboratories. We present a cost-effective nanofluidic-based immunosensor for low-noise real-time kinetic measurement of fluorescent-labelled protein binding. With the combination of fluorescence microscopy and reversed buffer flow operation, association and dissociation kinetics can be accessed in one single experiment without extra buffer loading step, which results in a simplified operation and reduced time of analysis compared to typical microfluidic immunoassays. Kinetic constants of two representative protein-ligand binding pairs (streptavidin/biotin; $\operatorname{IgG} / \mathrm{anti}-\mathrm{IgG}$ ) were quantified. The good agreement of extracted rate constants with literature values and analogous SPR measurements indicates that this approach is applicable to study protein interactions of medium- and high-affinities with a limit of detection down to $1 \mathrm{pM}$, regardless of the analyte size.
\end{abstract}

Keywords: Nanofluidics; Biosensors; Fluorescence microscopy; Interaction kinetics 


\section{Introduction}

Analysis of protein interactions provides a fundamental understanding of their physiological functions at the cellular scale in virtually all chemical and biological processes. These studies offer essential information for the development of improved diagnostic tools and new therapeutic treatments against diseases (Ideker and Sharan, 2008). Over the last few decades, affinity based biosensors, exploiting the interaction between a free target analyte and an immobilized receptor on a solid surface, have been a key solution in characterizing biospecific interactions in vitro. This method reveals the affinity and kinetic information of various binding events particularly protein-ligands and nucleic acids (Malmqvist and Karlsson, 1997; Nguyen et al., 2007). Affinity biosensor implemented on solid-state device that couple immunochemical reactions to a transducer is termed as immunosensor. Immunosensors can be divided into different categories depending on the detection principles, for example, optical, electrochemical, microgravimetric and thermometric (Luppa et al., 2001) schemes. Optical based immunosensors are the most widely used in clinical, food and environmental applications due to their performance characteristics.

Among optical based immunosensors, surface plasmon resonance (SPR) is currently the standard commercialized technology routinely used in the field of pharmaceutical and life sciences, offering a real-time detection of broad range of biomolecular interactions without labeling requirement (Myszka and Rich, 2000). Practically, measuring the interactions of lowmolecular weight analytes such as hormones, antibiotics, and chemical drug-fragments with SPR instrument is a key limitation in terms of sensitivity. Furthermore, if the reaction is too fast, with a high association rate, the obtained kinetic information is not reliable due to the strong effect of mass transfer (Nguyen et al., 2007). More importantly, high-cost dedicated sensor surfaces and integration of optical components are required, which in turn increases the overall assay costs and complicate the instrument setup.

Traditional fluorescence stopped-flow has been a method of choice for most biologists to study millisecond kinetic reactions of various biomolecular interactions in solution, for example, protein-DNA interactions (Esadze and Iwahara, 2014), ligand binding (Olsen et al., 1992) and enzyme reactions (Zhang et al., 2004). Total-internal-reflection fluorescence (TIRF) is a powerful analytical tool in visualizing real-time reversible interactions of fluorescent species on the surface with single-fluorophore sensitivity and minimized 
background fluorescence (Lakowicz, 2006; Schneckenburger, 2005). TIRF has been employed in combination with fluorescence polarization (FP) and fluorescence correlation spectroscopy (FCS) to determine affinities and reveal binding kinetics of fluorescently labeled IgG with mouse receptor FcgammaRII and human estrogen receptors with their specific ligands (Kwok and Cheung, 2010; Lieto et al., 2003), with nanoscale spatial resolution. Other fluorescence based techniques enabling real-time measurement of biomolecular recognition events include fluorescence after photo bleaching (FRAP) (Lele and Ingber, 2006) and Förster resonance energy transfer (FRET) (Li et al., 2007). On top of everything, Episentec has newly developed an add-on tool, the label-enhanced SPR technology, breaking the performance barriers of conventional SPR. This new technology aims at enhancing sensitivity and selectivity together with capability to analyze small molecules and fast kinetic reactions by using developed Episentec ${ }^{\mathrm{TM}}$ dye labels (Granqvist et al., 2013). These aforementioned examples have clearly proved that label-based techniques are still up-to-date and are readily applicable for investigating binding kinetics of ligand-receptor interactions in complement with label-free techniques to bring on biologically meaningful information.

In parallel, we have lately witnessed remarkable progress of novel micro- and nano-fluidic devices, allowing single molecule analysis (Dittrich and Manz, 2005; Yeh et al., 2012), cell sorting (Mazutis et al., 2013; Wang et al., 2005), DNA separation (Ashton et al., 2003; Fu et al., 2007), and fast multiplexed protein detection (Didar et al., 2012; Mitsakakis and Gizeli, 2011). The combination of nanofluidics with advanced biosensor technology paves new roads for point-of-care clinical diagnostics particularly owing to their capability to integrate such devices into lab-on-a-chip as the dimensions scale down. Nanofluidic-based biosensors predominately reduce the consumption of costly biological reagents with enhanced speed of analysis thanks to the shortened diffusion distance between immobilized probe molecules and flowing analytes in highly confined channels. First attempts to use biofunctionalized nanochannel for detection of streptavidin molecule using label-free impedance measurement in liquid induced flow has been reported (Karnik et al., 2005; Schoch et al., 2007). Though enhanced surface binding reactions have been demonstrated using pressure-driven flow over diffusion-limited reactions, long response time of 1-2 hours were required due to the lack of localized selective surface modifications (Schoch et al., 2007).

To address the issue of localized and selective surface modifications in nanochannels, we have developed a strategy using pre-functionalized nanofluidic channels with biocompatible encapsulation technique for DNA and protein detection (Leïchlé et al., 2012). We have shown 
that by confining the reaction volume into sub- $\mu \mathrm{m}$ thick channels, fluorescence conjugated biomolecules can be detected in real-time without cumbersome washing steps using a conventional fluorescence microscope (Leïchlé and Chou, 2015). This unique feature is specific to nanometer scale channels because the sample volume reduction during observation leads to negligible levels of fluorescence background, thus allowing us to directly probe the sensing surface without the need of using complicated and expensive setups, such as SPR, TIRFM, or quartz crystal microbalance (QCM). Moreover, the drastic reduction of diffusion length permits to operate in a reaction-limited regime with optimized target capture efficiency. Hence, all target molecules injected in the device are analyzed and because the typical sample volume is in the $\mathrm{pL}$ range, i.e. 4 orders of magnitude smaller than the sample volume of conventional microfluidic biosensor formats (Darain et al., 2009), this platform is especially suitable to the analysis of ultra-small samples, e.g. the content of few cells.

Here, we further demonstrate that this cost-effective sensing platform relying on the combinational use of biofunctional nanoslits and fluorescence detection, can achieve real-time monitoring of protein binding kinetics in physiological liquid environment with kinetic constant extraction, a platform we called Nanofluidic Fluorescence Microscopy (NFM). Fluorescence microscopy offers the possibility to carry out spatially resolved measurements where each camera pixel represents an independent measurement of the reaction kinetics. Hence, in addition to the high sensitivity with no analyte mass dependence inherent to this detection technique, large sampling area over a number of pixels ensures reduced statistical errors. On the other hand, because the fluorescent background level is proportional to the channel height, sub-micrometer channels offer signal to noise ratio of at least 100 on a large scale of dissociation constant $K_{D}$, in theory from the $\mathrm{pM}$ to the sub- $\mu \mathrm{M}$ range, that concerns most molecules of interests. Here, two representative protein-receptor pairs of different affinities, streptavidin/biotin (high affinity) and mouse IgG/anti-mouse IgG (medium affinity), were chosen to demonstrate the capability of our devices to determine kinetic parameters. Binding kinetics of streptavidin/biotin interaction was investigated in our study since monitoring this high affinity recognition with the standard SPR method has not yet been addressed. IgG/anti-IgG pair was chosen as a generic candidate model for other types of protein-ligand interactions.

Molecular interactions within biofunctionalized nanoslits were visualized by classical fluorescence microscope under pressure-driven flow (Fig. 1a). The results presented here show that our device was capable of generating full kinetic sensorgram including both 
association and dissociation phases in one single-experiment with a single injection via reversed buffer flow operation, which is another golden feature of our device (Fig. 1b). Indeed, because we operate in full target capture, all upcoming molecules injected onto the sensor are consumed by the reaction and a diffusing layer (depleted layer) forms at the sensor site. This depleted layer constitutes a clear boundary between regions where the concentration of analyte drops from maximum to zero. Thus, the liquid downstream the sensor is analyte free and the dissociation study can be simply implemented after completion of the association phase by reserving the fluid flow within the nanochannel instead of injecting new buffer in the inlet, which results in a simplified operating protocol and reduced time of analysis. The extraction of binding constants was conducted using a finite element model, an analytical method, and a commercial software package to demonstrate that our kinetic data could steadily be applied to various fitting approaches. To validate the performance of our proposed device for kinetic constant determination, the extracted on/off rates were compared with the one from literatures and with analogous SPR measurements.
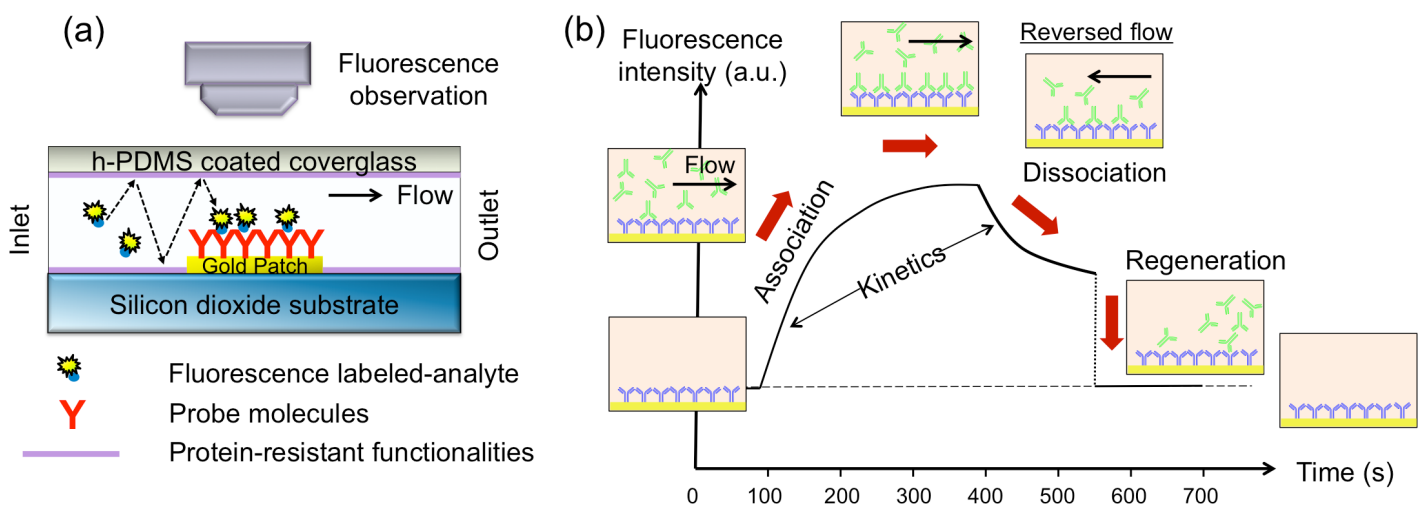

Fig. 1. (a) Schematic representation of biofunctional nanoslit used in protein kinetic study. Receptor probe molecules are immobilized on the gold sensor surface located at the bottom of the nanochannel. Fluorescence labeled target molecules are introduced from the inlet by means of pressure-driven flow. The kinetic reaction of protein-ligand binding is monitored in real-time using fluorescence microscopy. (b) Typical sensorgram of kinetic analysis performed in nanofluidic device. The dissociation phase is simple induced by reversing the fluid flow in order to replace the analyte solution by the buffer solution. 


\section{Material and methods}

\subsection{Surface chemistry}

For selective immobilization of the bio-receptors on the surface inside nanoslits, goldthiol chemistry was utilized via self-assemble monolayer (SAM) formation (Fig. 2). Gold surface was chosen for the local surface immobilization of bioreceptor probes in order to work with surfaces similar to the ones used in common SPR measurements. In this work, SAMs are composed of two thiol species, 1) hydroxyl-terminated thiol or a spacer and 2) biotinylated thiol used for further modification via biotin-streptavidin linkage. Optimization of the mixing ratio between these two compounds allows better control of immobilized surface probe densities on the sensor surface, which is the crucial parameter for kinetic studies. For the streptavidin-biotin interaction model, $1 \%$ of biotinylated thiol was used to obtain an optimum surface density of immobilized biotin to avoid the problem of non-steric hindrance upon binding which could change their kinetic binding behaviors. On the other hand, $10 \%$ biotinylated thiol was employed in the second model of mouse IgG/anti-mouse IgG interaction, providing the maximum density of immobilized streptavidin molecules for subsequent anchoring of the biotinylated anti-mouse IgG receptor. This surface chemistry strategy offers wide spread use of biotinylated proteins as the receptors for targeting other specific ligands.

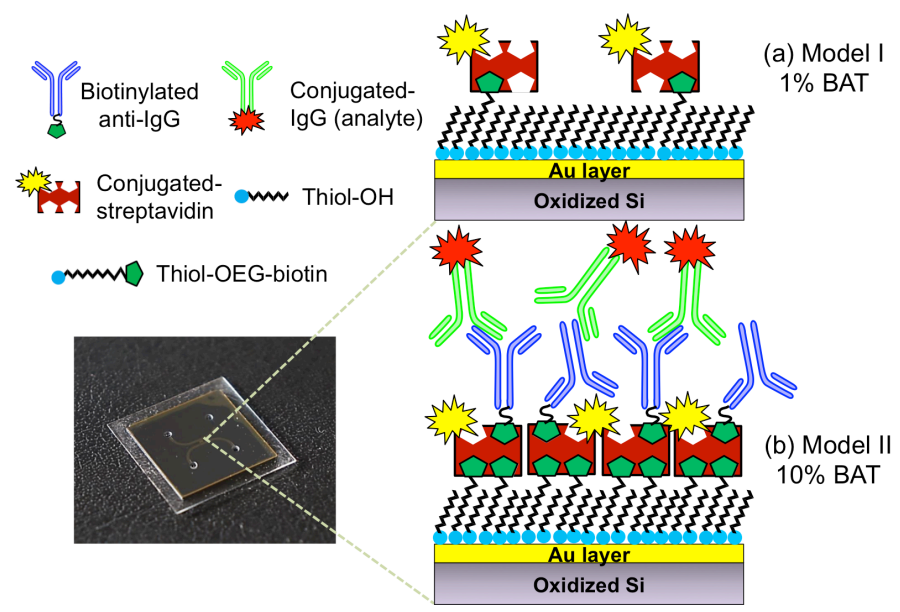

Fig. 2. Schematic illustrations (not to scale) of two surface functionalization architectures on gold sensors used for protein kinetic study in nanoslits. (a) Model I streptavidin-biotin interaction on $1 \%$ biotinylated thiol modified surface; (b) Model II mouse anti-rabbit $\mathrm{IgG} /$ anti-mouse $\mathrm{IgG}$ interaction via biotin-streptavidin linkage on $10 \%$ biotinylated thiol modified surface. A photograph of fabricated nanoslit device is also provided. 


\subsection{Fabrication of bio-functionalized device}

The fluidic device consists of a set of $450 \mathrm{~nm}$ deep, $50 \mu \mathrm{m}$ wide nanochannels binding two $5 \mu \mathrm{m}$ deep, $400 \mu \mathrm{m}$ wide microfluidic channels in a silicon substrate. The chips were fabricated using micromachining methods including conventional photolithography and reactive ion etching (details of the fabrication process are provided in S2 of the supplementary information).

The functionalization protocol used to graft the probe molecules onto the gold sensors embedded in the nanofluidic channels was partly carried out before chip sealing (see details in S3 of the supplementary information). This first functionalization step resulted in the coverage of the sensor surface with a mixed monolayer of hydroxy-terminated thiol/biotinterminated thiol, thus suitable for the streptavidin-biotin interaction studies but also allowing further modification after encapsulation. Encapsulation of the fabricated micro- and nanochannels was carried out by means of a hard-PDMS (h-PDMS) coated cover glass with oxygen plasma activation (S4 of supplementary information). After encapsulation step, the chips were filled with a blocking solution to prevent non-specific adsorption of proteins on the channel walls.

After chip bonding, surface functionalization was performed in flow to immobilize receptor molecules on the gold surface. First, streptavidin was grafted onto biotinylated SAM layer by introducing a $100 \mathrm{nM}$ solution of Alexa Fluor 488 conjugated streptavidin (ST-AF 488 ) at a constant flow velocity of $200 \mu \mathrm{m} / \mathrm{s}$ for $15 \mathrm{~min}$ until the saturation was achieved. After rinsing with the buffer solution, the biotinylated anti-mouse IgG receptor was subsequently immobilized onto the sensor via streptavidin-biotin linkage by injecting a 100 $\mathrm{nM}$ solution of biotinylated goat anti-mouse $\mathrm{IgG}$ for $15 \mathrm{~min}$ and rinsing with the buffer solution for $5 \mathrm{~min}$.

\subsection{Kinetic assay}

After the sensor was functionalized with the desired receptors, the fluorescently labeled analyte solution was introduced to the sensor embedded nanoslits. The sensor was monitored in real-time upon binding of the fluorescent target analyte to the corresponding surface immobilized probes using an inverted fluorescence microscope (see S6 of supplementary information). Reverse buffer operation was employed to achieve a full kinetic sensorgram (association and dissociation phases). The working principle of this operation is simple and 
straightforward. Briefly, the fluorescent-targeted analyte is injected from one inlet during the association phase for the binding reaction. The analyte flow is then stopped and the pressure on the opposite inlet is applied to allow the introduction of the fresh buffer solution for the dissociation of the surface bound complex. This operation can easily be accomplished by switching the automated pressure controller from one inlet to another one without having to remove the setup for refiling the new solution. This operation is key feature specific to our device in order to accomplish a full kinetic sensorgram in one single reagent loading, which is commonly impractical in case of classical microfluidic formats. Indeed, in the present nanoslit configuration, the thin diffusion layer is formed within the channel depth and propagates along the channel length (as opposed to microfluidic sensors, where the diffusion layer grows on top of the sensor in the depth of the channel); there is thus clear boundary between the analyte and the buffer and pressure-driven flow can be conveniently used to switch between the two phases.

\subsection{Data analysis and kinetic constant extraction}

Fluorescence images were properly analyzed to derive the normalized specific binding fluorescence intensities and plot the resulting sensorgram (see S6 of supplementary information). To extract the kinetic constants from binding assays, the sensor responses from various analyte concentrations were fitted with three different procedures. The first method involves use of a developed finite element model (FEM) to investigate the behavior of our devices and to predict the binding kinetics, regarding the convection-diffusion equations for mass transport of analyte species and the binding reactions between the analytes and the surface immobilized receptors (see S7 of supplementary information for details). Based on the calculated Damköhler numbers being less than one (see section 3.1), our pressure-driven flow based nanoslit biosensor is not limited by the mass transport of the analytes, which could significantly reduce the sensor performances in terms of sensitivity, response time and kinetic data accuracy. Therefore, our computational model is relatively similar to previous studies (Choi et al., 2010) rather than two-compartment models (Myszka et al., 1998). Our experimental data was fitted with the simulated kinetic curves by means of coefficient of determination (R-square). The second fitting approach is based on a simplified analytical solution called well-mixed model, assuming pseudo first order kinetics and that the kinetics are not mass-transfer limited (Karlsson et al., 1991). Finally, an available commercial BIAevaluation software package (GE Healthcare) was used to estimate the binding constants from our experimental kinetic data obtained in nanoslits. To this aim, separate fitting of 
association and dissociation phases (global-local fit) was performed with a Langmuir fit model.

\subsection{Validation with SPR setup}

To further validate the ability of our device for extraction of rate constants of biomolecular events, an analogous real-time kinetic assay was conducted to study the interaction of mouse IgG/anti-mouse IgG using surface plasmon resonance (SPR) setup (see S8 of supplementary information). The surface chemistry for receptor immobilization on the dedicated SPR sensor surface and the kinetic binding conditions were kept the same as the one used in nanoslits. Global analysis was performed to simultaneously fit the association and dissociation phases with one set of rate constants using BIAevaluation 4.1.1 software package. The kinetic parameters obtained from nanoslit experiments were then compared with that obtained from SPR measurements.

\section{Results and discussion}

\subsection{Regime of operation}

In order to assess the operation conditions of our nanofluidic embedded biosensor, it is essential to estimate the Peclet number $\left(\mathrm{Pe}_{H}\right)$ with respect to the channel height, the sensor size, and the Damköhler number $\left(D_{a}\right)$. The Peclet number relates the rate of convection to the rate of diffusion, $P e_{H}=U_{m} H / D$, where $U_{m}$ is the mean flow velocity (in the range of $200 \mu \mathrm{m} / \mathrm{s}$ for a typical experiment), $H$ is the channel height $(450 \mathrm{~nm})$, and $D$ is the diffusion coefficient $\left(7.4 \times 10^{-11} \mathrm{~m}^{2} / \mathrm{s}\right.$ for $53 \mathrm{kDa}$ streptavidin molecule (Spinke et al., 1993) and $1 \times 10^{-11} \mathrm{~m}^{2} / \mathrm{s}$ for $150 \mathrm{kDa}$ IgG molecule (Hu et al., 2007)) . The sensor size is expressed as $\lambda=L / H$, where $L$ is the sensor length (e.g. the whole sensor length or the length of 10 pixels used in data analysis). The Peclet numbers of 1.2 and 9 are found in case of streptavidin and mouse $\operatorname{IgG}$, respectively. These numbers being close to the unity imply that the time for the analyte to diffuse across the entire height of the channel is equal to the time for the analyte to be transported over the sensor via convection. Theoretically, all analyte molecules in the channel should have the possibility to interact with the probe on the sensor surface, and the system approaches the limit of full collection when $P e_{H}<0.5$ (Sjoelander and Urbaniczky, 1991). For both models, the sensor size $(\lambda)$ can be calculated as 111 for the whole sensor length $(50 \mu \mathrm{m})$ and 8.9 in case of 10 pixels data collection $(4 \mu \mathrm{m})$. These dimensionless numbers again describe our sensor to fall into "full collection" regime at sufficiently low $P e_{H}$ and large 
enough sensor $(\lambda)$ according to earlier work (Squires et al., 2008). On the other hand, the Damköhler number $\left(D_{a}\right)$ is expressed as the ratio between the rate of reaction and the rate of

diffusion, $D_{a}=\frac{k_{a} b_{m} H}{D}$, where $k_{a}$ is the association rate constant $\left(1 \times 10^{6} \mathrm{M}^{-1} \mathrm{~s}^{-1}\right.$ for streptavidinbiotin binding and $8 \times 10^{5} \mathrm{M}^{-1} \mathrm{~s}^{-1}$ for anti-mouse/mouse $\mathrm{IgG}$ interaction) and $b_{m}$ is the immobilized probe density $\left(4 \times 10^{-8} \mathrm{~mol} / \mathrm{m}^{2}\right.$ for streptavidin-biotin model and $1 \times 10^{-9} \mathrm{~mol} / \mathrm{m}^{2}$ for mouse $\operatorname{IgG}$ /anti-mouse $\operatorname{IgG}$ model). The calculated $D_{a}$ being smaller than one ( 0.24 for streptavidin-biotin and 0.036 for anti-mouse/mouse $\operatorname{IgG}$ ) indicates that kinetic reactions fall into a reaction- limited regime and not diffusion-limited.

\subsection{Kinetic experiments on Model I: Streptavidin/biotin}

Kinetic experiments were performed on biotinylated thiol modified nanochannel after confirming that pre-modified probe molecules maintained their bio-reactivity following the chip encapsulation procedure (see S5 of supplementary information). ST-AF 488 solutions ranging in concentration from $10 \mathrm{nM}$ to $100 \mathrm{nM}$ were introduced continuously at a constant flow velocity of $200 \mu \mathrm{m} / \mathrm{s}$ and allowed to bind with biotin immobilized on sensor surfaces. The fluorescence images were recorded in real-time to monitor the association process of this specific binding event. Due to very high binding affinity and exceptionally long dissociation time of streptavidin-biotin recognition system, four different devices were employed to measure the binding constants of streptavidin at each concentration.

The fluorescence intensities were normalized and fitted with simulated data attained from a finite element model. The relative sensor responses of streptavidin-biotin binding at various concentrations fitted with a simulation model are shown in Fig. 3a. It was observed that the experimental data of streptavidin binding at low concentration $(10-50 \mathrm{nM})$ fitted the simulation data well with R-square in a range of 0.99 . Nevertheless, high concentration of streptavidin $(100 \mathrm{nM})$ shows a relatively poor fit with the simulation results (R-square = 0.95). This might be due to several reasons pronounced at high analyte concentration including steric hindrance, heterogeneity of the surface, non-specific binding, deviation from 1:1 interaction model etc. To determine the binding affinity based on the computational model, the association and dissociation rate constants at each concentration were extracted from the best fit of the predicted binding curves to the experimental data. The average association rate constant $\left(k_{a}\right)$ was determined to be $7.1 \times 10^{5} \mathrm{M}^{-1} \mathrm{~s}^{-1}$ and dissociation rate constant $\left(k_{d}\right)$ of $8.0 \times 10^{-7} \mathrm{~s}^{-1}$ was observed from the best fit for all concentrations. Additionally, a lower off-rate was also applied in the simulation model, but there was no 
change in sensor responses. As a result, dissociation constant $\left(K_{D}\right)$ for streptavidin-biotin interaction can be estimated as $K_{D} \leq 1.1 \times 10^{-12} \mathrm{M}$.

The effect of various flow velocities ranging from 200-465 $\mu \mathrm{m} / \mathrm{s}$ (associated to the $P e_{H}$ number in the range of 1.2-2.8) on binding kinetics were also investigated however the sensor responses did not show any significant differences, indicating no mass transport limitation as expected. Therefore, the binding constant can also be determined using a simple "well-mixed" model, assuming a simple biomolecular first-order interaction. The binding of analyte to the sensor surface with an association rate constant $k_{a}$ and dissociation rate constant $k_{d}$ is given by

$$
C_{S}=\frac{k_{a} b_{m} C\left(1-e^{-\left(k_{a} C+k_{d}\right) t}\right)}{k_{a} C+k_{d}}
$$

where $C$ is the analyte concentration in bulk solution, $C_{s}$ is the bound complex concentration on the surface and $b_{m}$ is the probe density or binding sites on the surface. This model is defined as "well-mixed" model assuming that the detection kinetics are reaction rate-limited and the analyte concentration is constant and uniform everywhere above the sensor surface. The apparent time constant is given by $K_{a p p}=k_{a} C+k_{d}$, which can be derived by fitting the kinetic curves to equation 1. A plot of apparent time constant as a function of target analyte concentration is shown in Fig. 3b. It is seen that the apparent time constant increases with increasing analyte concentration. The plot gave a linear fit with a slope of 0.0003 and Rsquare of 0.9903. A derived slope of $0.0003 \mathrm{nM}^{-1} \mathrm{~s}^{-1}$ results in an association rate constant $k_{a}$ of $3.0 \times 10^{5} \mathrm{M}^{-1} \mathrm{~s}^{-1}$. This derived $k_{a}$ value is in a good agreement with the value extracted from our finite element model and within the reported range in the literatures based on microfluidic platform (Coltro et al., 2014; D’Agata et al., 2008; Srisa-Art et al., 2008), confirming the validity of the kinetic computational model to predict the binding responses of biospecific interactions. 
(a)

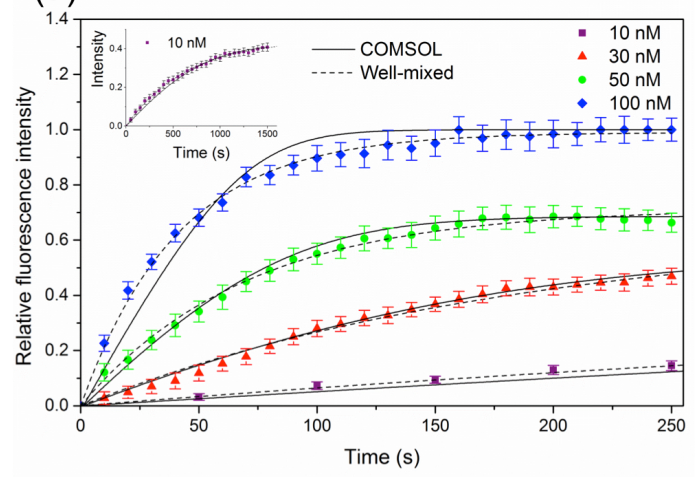

(b)

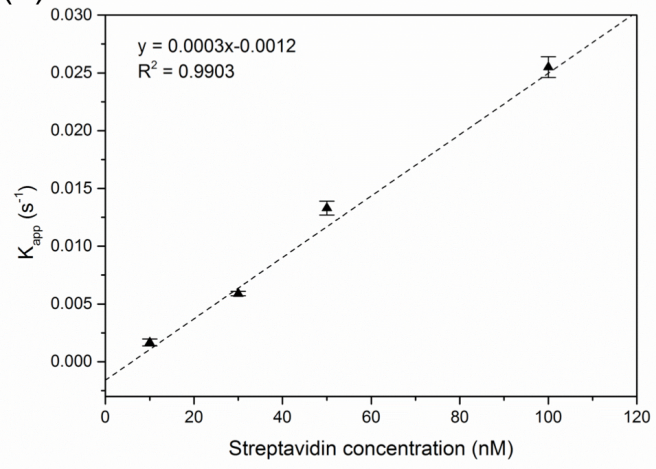

Fig. 3. (a) Real-time sensor responses of streptavidin-biotin interaction measured in biofunctional nanoslits. Kinetic curve obtained from each concentration is attributed to the measurement from a different nanofluidic device (the error bars: standard errors). The sensor responses were normalized to the relative fluorescence intensity and fitted with the computation model based on finite element method and well-mixed model assuming a simple Langmuir 1:1 interaction. The black (-) and (----) lines represent the predicted curves from the best fit of finite element simulation and well-mixed model, respectively. (b) Plot of apparent rate constants $\left(K_{a p p}\right)$ determined from non-linear least-squares fit as a function of streptavidin concentration. The plot shows linear trends with R-square value of 0.9903 .

\subsection{Kinetic experiments on Model II: Mouse IgG/anti-mouse IgG}

To further validate our kinetic model, mouse immunoglobulin G (IgG) and anti-mouse IgG was chosen as another protein-receptor pair. After chip encapsulation, the chip bearing immobilized biotinylated thiols was functionalized in flow with the fluorescently labeled streptavidin (ST-AF 488) followed by the attachment of biotinylated anti-mouse IgG receptors. For fluorescence imaging purpose, two different dyes with non-overlapping emission spectra were selected in order to distinguish between the immobilization process of streptavidin and the subsequent binding study between the non-fluorescent immobilized receptors and the fluorescently labeled IgG analytes.

Kinetic measurements were performed by introducing various concentrations of Alexa Fluor-647 conjugated mouse anti-rabbit IgG (mIgG-AF 647) target analyte (diluted in PBS buffer) ranging from 0.46 to $15 \mathrm{nM}$ to anti-mouse modified sensor chip under continuous flow velocity of $200 \mu \mathrm{m} / \mathrm{s}$. The bound mouse $\mathrm{IgG}$ was allowed to dissociate from the surface 
receptor by injection of a pure buffer solution via the reversed flow operation. The fluorescence images were recorded in real-time to monitor the association and dissociation processes inside nanoslit (Fig. 4a). In this study, the association time was fixed the same for all target concentrations enabling a global analysis using BIAevaluation software package. The sensor surface was regenerated by injecting the solution of $10 \mathrm{mM}$ glycine- $\mathrm{HCl} \mathrm{pH} 2.0$ for a short period of time and thoroughly rinsed with buffer solution before performing the next measurement cycle. Additionally, an irrelevant target analyte (Alexa Fluor-600 goat antirabbit $\mathrm{IgG}$ ) was introduced to anti-mouse immobilized nanoslit to verify non-specific interaction however no significant increase in fluorescence intensity was observed.

The resulting kinetic data were then normalized and fitted with three different models: i) our finite element simulation, ii) the well-mixed model and iii) the global-local fitting by BIAevalulation software (Fig. 4b). Based on finite element computational modeling, the binding rate constants were determined from the best fit of the predicted kinetic curves to the experimental data by means of coefficient of determination. It can be observed that our simulated kinetic model describes the immunoreaction assay in nanoslit well as each experimental binding curve was fitted accurately (R-square in a range of 0.99) (Fig. S5). The average association rate constant $\left(k_{a}\right)$ was determined to be $8.0 \times 10^{5} \mathrm{M}^{-1} \mathrm{~s}^{-1}$ and dissociation rate constant $\left(k_{d}\right)$ of $6.2 \times 10^{-4} \mathrm{~s}^{-1}$ was achieved, giving an equilibrium dissociation constant $K_{D}$ of $0.77 \mathrm{nM}$. Subsequently, the experimental data were fitted to the 'well-mixed' analytical model with non-linear least squares method and the apparent time constant $\left(K_{\text {app }}\right)$ was plotted as a function of analyte concentration, giving a good fit with R-square of 0.9959 (Fig. 4c). The desorption of bound analyte from the surface receptor was estimated using a first-order exponential decay. This results in the association and dissociation rate constants of $6.0 \times 10^{5}$ $\mathrm{M}^{-1} \mathrm{~s}^{-1}$ and $5.5 \times 10^{-4} \mathrm{~s}^{-1}$, respectively. Finally, the kinetic data were examined using a globallocal analysis from BIAevaluation software. Comparable to our simulation, the best fit was found using a Langmuir 1:1 binding model giving residual versus time plots lower than 0.1 (Fig. 4b). The average values of $k_{a}$ and $k_{d}$ were determined to be $12 \times 10^{5} \mathrm{M}^{-1} \mathrm{~s}^{-1}$ with SE of $2.0 \times 10^{4}$ and $5.0 \times 10^{-4} \mathrm{~s}^{-1}$ with SE of $1.8 \times 10^{-5}$, respectively, supporting the validity of our finite element model for good quantitative description of the kinetic data. 
(a)

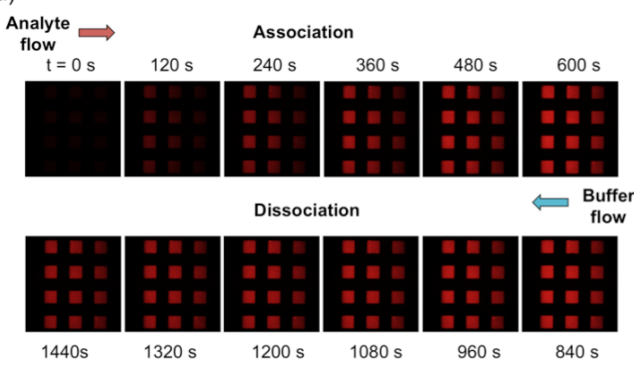

(b)
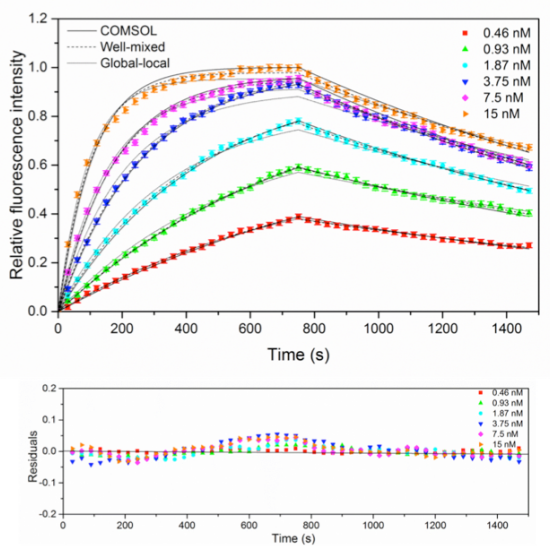

(c)

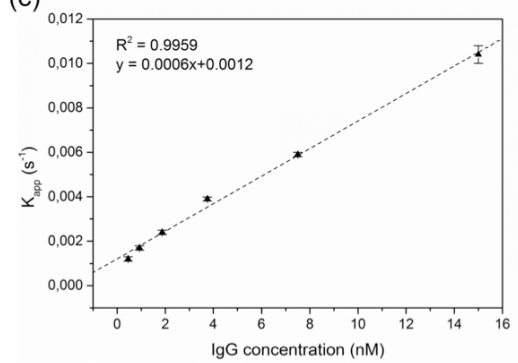

(d)
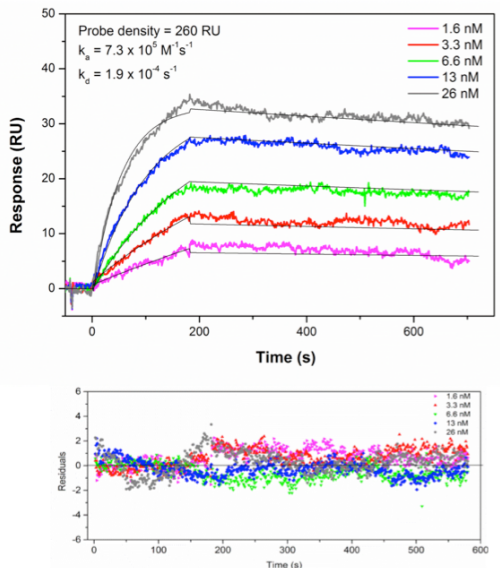

Fig. 4. (a) Fluorescence image snapshots taken during association and dissociation processes upon Alexa Fluor-647 mouse anti-rabbit IgG (1.87 nM) injection into anti-mouse modified nanoslit. (b) Real-time sensor responses at various analyte concentrations ranging from 0.46 $\mathrm{nM}$ to $15 \mathrm{nM}$ (the error bars: standard errors). The data were normalized to relative fluorescence intensity and fitted with three different models: (1) finite element simulation, (2) well-mixed model and (3) global-local fitting by BIAevaluation software package, assuming a simple Langmuir 1:1 interaction. The black (-), (---) and (…) lines represent the predicted curves from the best fit of finite element simulation, well-mixed model, and global-local fitting, respectively. The residual versus time plots are also shown for all concentrations. (c) Plot of apparent rate constants $\left(K_{a p p}\right)$ as a function of mouse $\operatorname{IgG}$ concentration. (d) Sensorgrams of mouse IgG/anti-mouse IgG binding kinetics obtained from analogous SPR measurement. Kinetic curves were fitted using a 1:1 global analysis model from BIAevaluation software package (black line). The residual versus time plots are demonstrated for all concentrations. 


\subsection{Benchmarking with SPR}

To further evaluate our platform, using the same preparation coated-gold surface as nanoslits, an analogous real-time kinetic measurement of mouse IgG/anti-mouse IgG was conducted using surface plasmon resonance setup. Sensorgrams containing kinetic information of these binding events at various concentrations ranging from 1.6 to $26 \mathrm{nM}$ are shown in Fig. 4d. Global analysis was utilized to identify the binding model and quantify kinetic parameters using BIAevaluation software package. The best fit for the entire data was found with a 1:1 Langmuir binding model without influence of mass transport, giving $k_{a}$ and $k_{d}$ of $7.3 \times 10^{5} \mathrm{M}^{-1} \mathrm{~s}^{-1}$ with SE of $3.0 \times 10^{3}$ and $1.9 \times 10^{-4} \mathrm{~s}^{-1}$, with SE of $8.0 \times 10^{-6}$, respectively. Furthermore, repeated kinetic measurements were also conducted at different immobilized surface probe densities (Fig. S6) and with unconjugated target molecules (Fig. S7) to ensure the accuracy of determined kinetic parameters from the binding data and to investigate the influence of labeling on binding kinetics of molecular interactions. The estimated binding rate constants were still consistent. In summary, our nanoslit based biosensor platform yielded kinetic constants that are in a good agreement with the SPR measurement and in the range with literature values (Table 1), thus extending the validity of our platform for accurately quantifying the binding kinetics of biomolecular interactions. 
Table 1. Comparison of kinetic constants for streptavidin-biotin and IgG/anti-IgG binding between our biofunctional nanofluidic platform, SPR and literature

\begin{tabular}{lccc}
\hline $\begin{array}{l}\text { Biosensor platform } \\
\text { Model I: }\end{array}$ & Association rate & Dissociation rate & $\begin{array}{c}\text { Equilibrium } \\
\text { dissociation }\end{array}$ \\
Streptavidin-biotin & $\left(k_{a}, 10^{5} \mathrm{M}^{-1} \mathrm{~s}^{-1}\right)$ & $\left(k_{d}, 10^{-7} \mathrm{~s}^{-1}\right)$ & $\left(K_{D}, 10^{-12} \mathrm{M}\right)$ \\
\hline Nanoslit biosensor & & & \\
-FEM simulation model & $7.1 \pm 2.5$ & $8.0 \pm 1.2$ & $\leq 1.1$ \\
-Well-mixed model & $3.0 \pm 0.3$ & $\mathrm{~N} / \mathrm{A}$ & \\
\hline Literature (Coltro et al., & $4.4-450$ & 0.2 & $\sim 0.1$
\end{tabular}

2014; D’Agata et al., 2008;

Hosokawa et al., 2006;

Srisa-Art et al., 2008)

\begin{tabular}{lccc}
\hline Biosensor platform & Association rate & Dissociation rate & Equilibrium \\
Model II: & & & dissociation \\
IgG/anti-mouse IgG & $\left(k_{a}, 10^{5} \mathrm{M}^{-1} \mathrm{~s}^{-1}\right)$ & $\left(k_{d}, 10^{-4} \mathrm{~s}^{-1}\right)$ & $\left(K_{D}, 10^{-10} \mathrm{M}\right)$
\end{tabular}

\section{$\underline{\text { Nanoslit biosensor }}$}

$\begin{array}{llll}\text {-FEM simulation model } & 8.0 \pm 1.2 & 6.2 \pm 0.3 & 7.7\end{array}$

$\begin{array}{llll}\text {-Well-mixed model } & 6.0 \pm 0.3 & 5.5 \pm 0.4 & 9.2\end{array}$

$\begin{array}{llll}\text {-Global local fit } & 12 \pm 0.2 & 5.0 \pm 0.2 & 4.2\end{array}$

$\underline{\text { SPR }}$

$\begin{array}{llll}\text { (Global analysis) } & 7.3 \pm 0.1 & 1.9 \pm 0.1 & 2.6\end{array}$

\begin{tabular}{llll}
\hline Literature (Lee et al., 2007; & $2.5-13$ & 3.0 & $1.5-12$
\end{tabular}


Pereira et al., 2011; Piran

and Riordan, 1990; Yu et al., 2005) 


\subsection{Range of operation and detection limit}

The accurate determination of association and dissociation constants for the two couples of ligands presented here (streptavidin/biotin and mouse IgG/anti-mouse IgG) illustrates the various affinity strengths that can be studied with our system. Affinity studies usually require kinetic plots for several analyte concentrations ranging from $1 / 10$ to $10 K_{D}$ in order to adequately extract kinetics constants. Limitation of our technique in terms of working concentrations is given by the fluorescence signal to noise ratio, e.g. the fluorescence emitted by the analytes specifically interacting at the sensor surface $v$. the fluorescence due to the molecules flowing in the fluidic channel. In reaction-limited regime at equilibrium, for concentrated $\left(C>>K_{D}\right)$ and diluted solutions $\left(C<<K_{D}\right)$, where analyte molecules bind all or a fraction of the receptor molecules, we respectively have:

$$
\begin{aligned}
& S /_{N}=\frac{b_{m}}{H \times C} \\
& S /_{N}=\frac{b_{m}}{H \times K_{D}}
\end{aligned}
$$

Thus, the sampling volume reduction during observation translates into a decreased background with a signal to noise ratio inversely proportional to the channel height. Using the grafted probe density, $b_{m}$, obtained with the presented surface functionalization protocol for IgG grafting, the previous equations demonstrate that high enough $S / N$ can be obtained with sub-micrometer channels $(H<1 \mu \mathrm{m})$ even for medium to low affinity molecules, e.g. we can expect $S / N$ of $\sim 100$ in $100 \mathrm{~nm}$ deep channels for $K_{D}$ of $0.1 \mu \mathrm{M}$. In fact, the pM to the sub- $\mu \mathrm{M}$ range can be addressed with the presented technique, which concerns most molecules of interests.

Finally, to ensure that our device can be employed not only for investigating binding kinetics of protein-ligand interactions but can also be applied as a highly sensitive and rapid on-chip immunosensor, limit of detection (LOD) is another important performance characteristic for validation. In this study, the detection limit was determined by introducing different concentrations of mIgG-AF 647 target analyte solutions to anti-mouse IgG-modified nanoslit and the fluorescence intensity was recorded. The fluorescence intensity changes with the introduction of target analyte as a function of time are plotted in Fig. S8. It should be noted that the obtained LOD depends strongly on the sensitivity of the camera in which the imaging parameters were adjusted in our case to optimize this LOD. No significant increase in 
fluorescence intensity was observed when an analyte-free buffer solution was injected, while introduction of $1 \mathrm{pM}$ resulted in a significant increase in fluorescence intensity after $30 \mathrm{~min}$ $(S / N \sim 3)$. With a sample volume of $10.5 \mathrm{~nL}$, a mole detection limit can be determined as 10 zeptomole. Notably, increasing the analyte concentration to $10 \mathrm{pM}$, our sensor can easily detect the binding response signal within $5 \mathrm{~min}$. The value of detection limit obtained from our system for mouse IgG sensing is significantly better ( 100 times) than or comparable to existing microfluidic immunosensing formats (Ivnitski et al., 1998; Sapsford et al., 2001; Yu et al., 2005). Additionally, this on-chip immunosensor shows good repeatability with a standard deviation of $2.28 \%$ at analyte concentration of $5 \mathrm{nM}$ (Fig. S9). Furthermore, the total immunoassay time (including injection, immobilization and detection steps) required for mouse IgG detection based on streptavidin-biotin linkage for anti-mouse IgG immobilization was found to be approximately $40 \mathrm{~min}$ in our study while the traditional enzyme-linked immunosorbent assay (ELISA) takes hours to days to complete the whole assay. Therefore, our approach also demonstrates fast and sensitive immunosensing platform for protein detection with low reagent consumption ( 1000 times less than SPR setup), indicating a great potential in clinical diagnosis.

\section{Conclusions}

We have presented the first effective platform for real-time kinetic monitoring of protein interactions using nanofluidic-based immuno-biosensors coupled with conventional fluorescence microscopy and pressure driven flow. Remarkably, we have demonstrated that association and dissociation kinetics of immunoreaction occurred in nanoslit can be achieved in one-single experiment without removing the fluidic setup via reversed buffer flow technique which is impractical for classical microfluidic immunoassay formats. We have also validated that our kinetic data obtained from nanoslit platform can be readily applied to various fitting approaches, including the finite element model, the analytical model and the commercial software, to quantify kinetic parameters (on/off rates) of two protein-receptor binding pairs. The extracted values are consistent with SPR measurement and previously reported values in literatures. As a result, our new approach holds promise as an alternative tool for real-time detection with signal to noise ratio of at least 100 for a broad range of protein-ligand interactions with $K_{D}$ from $\mathrm{pM}$ to sub- $\mu \mathrm{M}$. Our platform is expected to achieve the level of performance of commercial SPR biosensor, with the additional advantages of 
simplicity, low sample consumption, optimized target capture, reduced statistical errors, no limitation of analyte size, and limit of detection down to the picomolar range without using any combined preconcentration or enzymatic amplification schemes. Furthermore, we believe our novel method provides a potential possibility to integrate into lab-on-a-chip with fast and multiplexed sensing for point-of-care clinical diagnostic and drug screening applications.

\section{Acknowledgements}

The authors acknowledge the Programme Blanc jointly sponsored by the Agence Nationale de la Recherche (ANR-13-IS10-0001) and the Ministry of Science and Technology, Taiwan (103-2923-M-001-007-MY3), and the MAE (Ministère des Affaires Etrangères) for the Doctoral Fellowship of P.T. This work was partly supported by the French RENATECH network, Academia Sinica Integrated Thematic Project (AS-103-TP-A01), and AOARD grant $\# 124002$.

\section{References}

Ashton, R., Padala, C., Kane, R.S., 2003. Microfluidic separation of DNA. Curr. Opin. Biotechnol. 14, 497-504.

Choi, C.J., Belobraydich, A.R., Chan, L.L., Mathias, P.C., Cunningham, B.T., 2010. Comparison of label-free biosensing in microplate, microfluidic, and spot-based affinity capture assays. Anal. Biochem. 405, 1-10.

Coltro, W.K.T., Neves, R. de S., Motheo, A. de J., da Silva, J.A.F., Carrilho, E., 2014. Microfluidic devices with integrated dual-capacitively coupled contactless conductivity detection to monitor binding events in real time. Sens. Actuators B Chem. 192, 239-246.

D’Agata, R., Grasso, G., Spoto, G., 2008. Real-Time Binding Kinetics Monitored with Surface Plasmon Resonance Imaging in a Diffusion-Free Environment. Open Spectrosc. J. 2, 1-9.

Darain, F., Gan, K.L., Tjin, S.C., 2009. Antibody immobilization on to polystyrene substrate-on-chip immunoassay for horse IgG based on fluorescence. Biomed. Microdevices 11, 653-661.

Didar, T.F., Foudeh, A.M., Tabrizian, M., 2012. Patterning multiplex protein microarrays in a single microfluidic channel. Anal. Chem. 84, 1012-1018. 
Dittrich, P.S., Manz, A., 2005. Single-molecule fluorescence detection in microfluidic channels--the Holy Grail in muTAS? Anal. Bioanal. Chem. 382, 1771-1782.

Esadze, A., Iwahara, J., 2014. Stopped-flow fluorescence kinetic study of protein sliding and intersegment transfer in the target DNA search process. J. Mol. Biol. 426, 230-244.

Fu, J., Schoch, R.B., Stevens, A.L., Tannenbaum, S.R., Han, J., 2007. A patterned anisotropic nanofluidic sieving structure for continuous-flow separation of DNA and proteins. Nat. Nanotechnol. 2, 121-128.

Granqvist, N., Hanning, A., Eng, L., Tuppurainen, J., Viitala, T., 2013. Label-Enhanced Surface Plasmon Resonance: A New Concept for Improved Performance in Optical Biosensor Analysis. Sensors 13, 15348-15363.

Hosokawa, K., Omata, M., Sato, K., Maeda, M., 2006. Power-free sequential injection for microchip immunoassay toward point-of-care testing. Lab. Chip 6, 236-241.

Hu, G., Gao, Y., Li, D., 2007. Modeling micropatterned antigen-antibody binding kinetics in a microfluidic chip. Biosens. Bioelectron. 22, 1403-1409.

Ideker, T., Sharan, R., 2008. Protein networks in disease. Genome Res. 18, 644-652.

Ivnitski, D., Wolf, T., Solomon, B., Fleminger, G., Rishpon, J., 1998. An amperometric biosensor for real-time analysis of molecular recognition. Bioelectrochem. Bioenerg. $45,27-32$.

Karlsson, R., Michaelsson, A., Mattsson, L., 1991. Kinetic analysis of monoclonal antibodyantigen interactions with a new biosensor based analytical system. J. Immunol. Methods 145, 229-240.

Karnik, R., Castelino, K., Fan, R., Yang, P., Majumdar, A., 2005. Effects of Biological Reactions and Modifications on Conductance of Nanofluidic Channels. Nano Lett. 5, $1638-1642$.

Kwok, K.-C., Cheung, N.-H., 2010. Measuring binding kinetics of ligands with tethered receptors by fluorescence polarization and total internal reflection fluorescence. Anal. Chem. 82, 3819-3825.

Lakowicz, J.R., 2006. Principles of Fluorescence Spectroscopy, 3rd ed. Springer, New York.

Lee, K.-H., Su, Y.-D., Chen, S.-J., Tseng, F.-G., Lee, G.-B., 2007. Microfluidic systems integrated with two-dimensional surface plasmon resonance phase imaging systems for microarray immunoassay. Biosens. Bioelectron. 23, 466-472.

Leïchlé, T., Chou, C.-F., 2015. Biofunctionalized nanoslits for wash-free and spatially resolved real-time sensing with full target capture. Biomicrofluidics 9, 034103. 
Leïchlé, T., Lin, Y.-L., Chiang, P.-C., Hu, S.-M., Liao, K.-T., Chou, C.-F., 2012. Biosensorcompatible encapsulation for pre-functionalized nanofluidic channels using asymmetric plasma treatment. Sens. Actuators B Chem. 161, 805-810.

Lele, T.P., Ingber, D.E., 2006. A mathematical model to determine molecular kinetic rate constants under non-steady state conditions using fluorescence recovery after photobleaching (FRAP). Biophys. Chem. 120, 32-35.

Lieto, A.M., Cush, R.C., Thompson, N.L., 2003. Ligand-receptor kinetics measured by total internal reflection with fluorescence correlation spectroscopy. Biophys. J. 85, 3294 3302.

Li, Y., Augustine, G.J., Weninger, K., 2007. Kinetics of complexin binding to the SNARE complex: correcting single molecule FRET measurements for hidden events. Biophys. J. 93, 2178-2187.

Luppa, P.B., Sokoll, L.J., Chan, D.W., 2001. Immunosensors--principles and applications to clinical chemistry. Clin. Chim. Acta. 314, 1-26.

Malmqvist, M., Karlsson, R., 1997. Biomolecular interaction analysis: affinity biosensor technologies for functional analysis of proteins. Curr. Opin. Chem. Biol. 1, 378-383.

Mazutis, L., Gilbert, J., Ung, W.L., Weitz, D.A., Griffiths, A.D., Heyman, J.A., 2013. Singlecell analysis and sorting using droplet-based microfluidics. Nat. Protoc. 8, 870-891.

Mitsakakis, K., Gizeli, E., 2011. Multi-sample acoustic biosensing microsystem for protein interaction analysis. Biosens. Bioelectron. 26, 4579-4584.

Myszka, D.G., He, X., Dembo, M., Morton, T.A., Goldstein, B., 1998. Extending the range of rate constants available from BIACORE: interpreting mass transport-influenced binding data. Biophys. J. 75, 583-594.

Myszka, D.G., Rich, R.L., 2000. Implementing surface plasmon resonance biosensors in drug discovery. Pharm. Sci. Technol. Today 3, 310-317.

Nguyen, B., Tanious, F.A., Wilson, W.D., 2007. Biosensor-surface plasmon resonance: quantitative analysis of small molecule-nucleic acid interactions. Methods 42, 150 161.

Olsen, K., Svensson, B., Christensen, U., 1992. Stopped-flow fluorescence and steady-state kinetic studies of ligand-binding reactions of glucoamylase from Aspergillus niger. Eur. J. Biochem. 209, 777-784.

Pereira, A.T., Novo, P., Prazeres, D.M.F., Chu, V., Conde, J.P., 2011. Heterogeneous immunoassays in microfluidic format using fluorescence detection with integrated amorphous silicon photodiodes. Biomicrofluidics 5, 14102. 
Piran, U., Riordan, W.J., 1990. Dissociation rate constant of the biotin-streptavidin complex. J. Immunol. Methods 133, 141-143.

Sapsford, K.E., Liron, Z., Shubin, Y.S., Ligler, F.S., 2001. Kinetics of antigen binding to arrays of antibodies in different sized spots. Anal. Chem. 73, 5518-5524.

Schneckenburger, H., 2005. Total internal reflection fluorescence microscopy: technical innovations and novel applications. Curr. Opin. Biotechnol. 16, 13-18.

Schoch, R.B., Cheow, L.F., Han, J., 2007. Electrical Detection of Fast Reaction Kinetics in Nanochannels with an Induced Flow. Nano Lett. 7, 3895-3900.

Sjoelander, S., Urbaniczky, C., 1991. Integrated fluid handling system for biomolecular interaction analysis. Anal. Chem. 63, 2338-2345.

Spinke, J., Liley, M., Schmitt, F.-J., Guder, H.-J., Angermaier, L., Knoll, W., 1993. Molecular recognition at self-assembled monolayers: Optimization of surface functionalization. J. Chem. Phys. 99, 7012-7019.

Squires, T.M., Messinger, R.J., Manalis, S.R., 2008. Making it stick: convection, reaction and diffusion in surface-based biosensors. Nat. Biotechnol. 26, 417-426.

Srisa-Art, M., Dyson, E.C., deMello, A.J., Edel, J.B., 2008. Monitoring of real-time streptavidin-biotin binding kinetics using droplet microfluidics. Anal. Chem. 80, $7063-7067$.

Wang, M.M., Tu, E., Raymond, D.E., Yang, J.M., Zhang, H., Hagen, N., Dees, B., Mercer, E.M., Forster, A.H., Kariv, I., Marchand, P.J., Butler, W.F., 2005. Microfluidic sorting of mammalian cells by optical force switching. Nat. Biotechnol. 23, 83-87.

Yeh, J.-W., Taloni, A., Chen, Y.-L., Chou, C.-F., 2012. Entropy-Driven Single Molecule Tugof-War of DNA at Micro-Nanofluidic Interfaces. Nano Lett. 12, 1597-1602.

Yu, C.-J., Chou, C., Hsu, H.-Y., Chan, T.-S., Lee, Z.-Y., Wu, H.-T., 2005. Fiber optic biosensor for monitoring protein binding kinetics. Proc. SPIE 5691, 200-208.

Zhang, Z., Rajagopalan, P.T.R., Selzer, T., Benkovic, S.J., Hammes, G.G., 2004. Singlemolecule and transient kinetics investigation of the interaction of dihydrofolate reductase with NADPH and dihydrofolate. Proc. Natl. Acad. Sci. USA 101, 2764 2769. 